\title{
Visualization software for Hydrophobic-polar protein folding model
}

\author{
R. Mavrevski ${ }^{1}$, M. Traykov² \\ University Center for Advanced Bioinformatics Research, Bulgaria \\ ${ }^{1}$ ORCID: 0000-0002-6968-8937, radoslav sm@abv.bg \\ 2 ORCID: 0000-0003-2507-1924, metodi_043@abv.bg
}

\begin{abstract}
The simplest and most used models of protein folding is the Hydrophobic-Polar (HP) model. The HP model labeling the amino acids as Hydrophilic (H) or Polar/ydrophilic (P). The folding of amino acids sequence is configured as self-avoiding walks on the $2 \mathrm{D}$ or $3 \mathrm{D}$ lattice, where the optimal conformation has maximum number of contacts between $\mathrm{H}$ amino acids ( $\mathrm{H}-\mathrm{H}$ contacts) that are not adjustment in amino acid sequence. In this paper, we develop and present software for visualization of HP protein folding problem under the HP model on the 2D square lattice. For the development of HP folding visualization software, we used MS Visual Studio, .NET Framework 2.0 and C\# language. If we have HP sequence and folding results for this sequence, obtained by optimization software as CPLEX or GUROBI, then using the $2 \mathrm{D}$ visualization software we can visualize the obtained results in square lattice. This visualization software is a valuable tool for the study of HP folding and is a great pedagogic instrument. All figures of HP folding included in this paper are actual screenshots of our visualization program.
\end{abstract}

Keywords: protein folding, HP model, visualization.

\section{Introduction}

The proteins are major and important class of biological macromolecules, which perform structural and catalytic functions in the muscle, skin, hair, etc. The protein is a chain where each element is one of 20 different amino acids [1-3]. An important property of proteins is their hydrophobicity, which is expressed in how much they are repel by the water. Understanding the processes leading to a protein folding into its functional state is the important problem in molecular biophysics. This determines how the protein chain folds into a complex conformation (conformation of the molecule). This conformation is stable, has small free energy and is very important for the function of the protein. It is important to note that the amino acid sequence is the primary structure. In the literature there are described different types of algorithms to predict the tertiary structure of proteins [4]. All these algorithms use an abstract model of real proteins, describing their characteristics. Most common models are the lattice models, which use a $2 \mathrm{D}$ or $3 \mathrm{D}$ lattices to describe the positions of the amino acids and an energy function that needs to be minimized according to positions of the amino acids in the lattice. This is the so-called Protein folding problem. The key model to solve the Protein folding problem is Dill's Hydrophobic-Polar (HP) model (so-called HP folding problem) that tries to form hydrophobic core, i.e. tries to put hydrophobic amino acid in the center of obtained fold surrounded by polar amino acid [5-9].

In the literature, there are many mathematical models described and algorithms for Protein folding problem or 
HP folding problem. However, the most articles describing mathematical models and algorithms for HP folding lack mentioning the visualization of the obtained results (folds). In this paper, we propose software for visualization of received folds in HP folding problem under 2D square lattice after we solve the problem using some optimization software, as a CPLEX or GUROBI. In the section 3 (Results) we present our experiments and results. Thus, it is possible to make detail analysis of the folding process, e.g. by comparison of different conformations for one input HP sequence or identifying the connectivity between conformations. Visualizing the folding paths (so-called selfavoiding paths), we can study different regions of the protein conformation, as well as determine the difficulty in folding of different proteins. Visualizing the folding is significant challenge owing to the similarity to nature state of the proteins. The other motivation to investigate and visualize the protein folding process in details is the potential help in understanding the role of protein functions $[7,8]$.

\section{Methods}

\subsection{Input files}

To solve the Protein folding problem in $2 \mathrm{D}$ or $3 \mathrm{D}$ HP model we generate .lp file (https://www.ibm.com/support/knowl edge center/SSSA 5P 12.5.0 /ilog.odms.cplex.help/CPLEX/FileFor mats/topics/LP.html) that contains description of a mathematical model for the problem. After that, we submit the obtained .lp file to MIP (Mixed Integer Programming) solver like CPLEX (https://www.ibm.com/analytics/cplex -optimizer) or GUROBI (http://www.gurobi.com/) and run the solver. When the solver finishes, we obtain a log file that contains solution to the mathematical model (see Figure 1). The obtained log file can be stored in different formats (according to the model description in .lp file), i.e. we do not know the format of the file so we transform the log file manually (using Notepad++ software) to the appropriate input file (Figure 2) with the ".txt" extension (named "flat_test.txt") for our visualization software. In the future, we plan to automate the transformation process.

The first column of the transformed file contains the variable names and the corresponding direction of movement ( $\mathrm{r}$ - right, $\mathrm{l}$ - left, $\mathrm{u}$ - upper, $\mathrm{d}$ - down) during the folding process. The second column contains solution value for the corresponding variable. In addition, we prepare manually (using Notepad++ software) second input file for our HP Folding Visualization software (named "flat_test_HP.txt") containing the HP sequence (see Figure 3). 


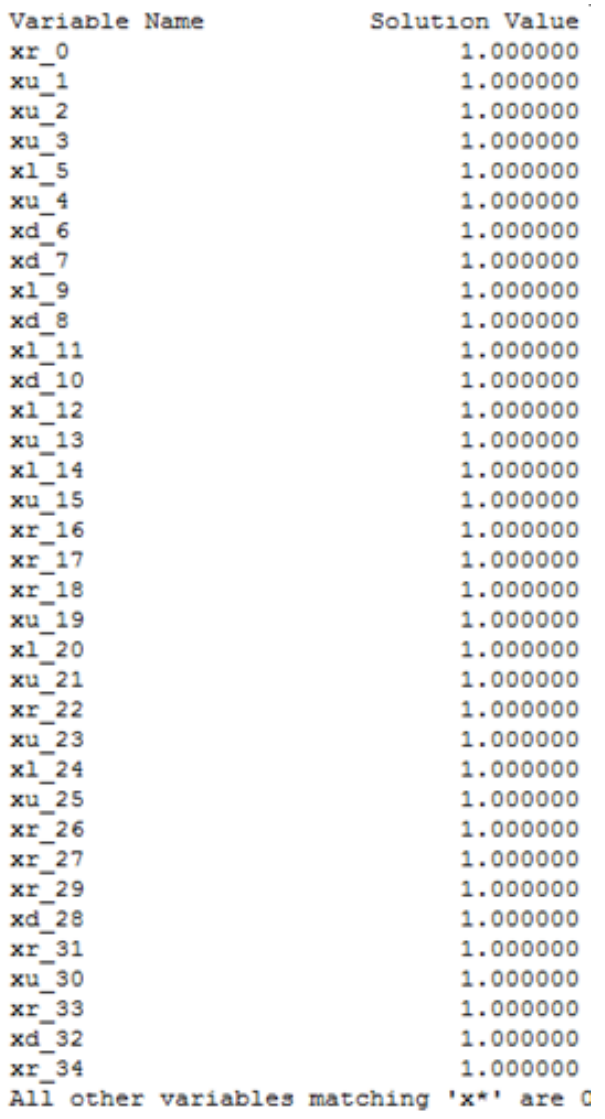

(a)

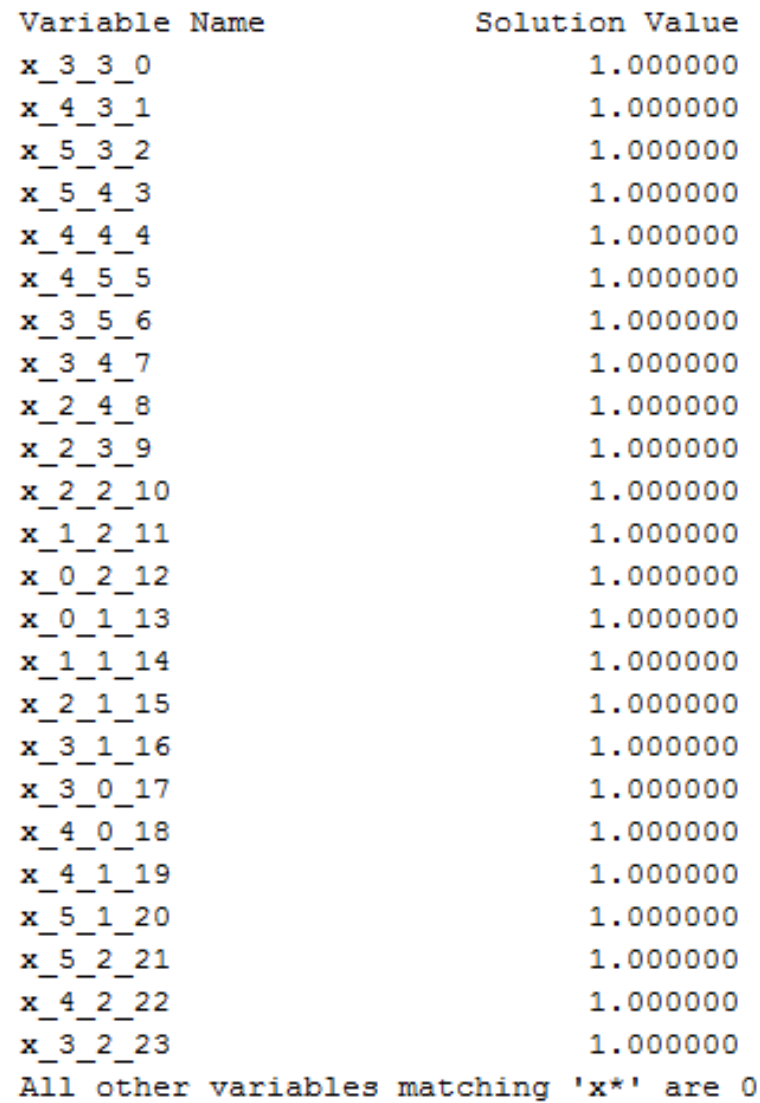

(b)

Fig. 1. Sample optimization software output log files: (a) sample 1, (b) sample 2

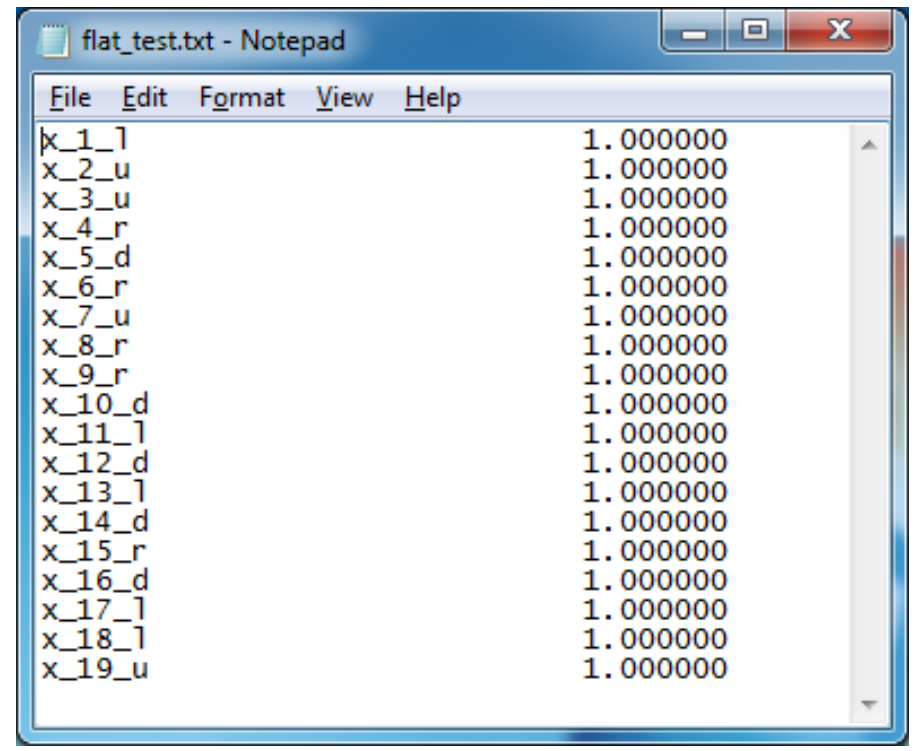

Fig. 2. Input file "flat_test.txt" for software "HP Folding Visualization"

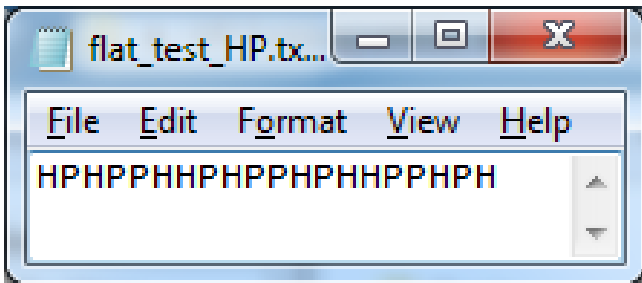

Fig. 3. Input file "flat_test_HP.txt" for software "HP Folding Visualization" 


\subsection{Development of visualization software}

In this article, we show software called "HP Folding Visualization" developed by us (see Figure 4 and 5). The aim of this software is to make visual representation of performers of HP protein folding in 2D lattice. We can run our software as desktop application, i.e. we do not need to make installation, just copy and paste the folder with software files on our computer. To develop the "HP Folding Visualization" software we use MS Visual Studio, .NET Framework 2.0 and C\# language. To create a simple and flexible grid to use in all cases where it is necessary to visualize HP folding process we used additional library

"SourceGrid.dll" (https://archive.codeplex.com/?p=sou rcegrid). There are available many controls of this type, but they are expensive, difficult to customize or not compatible with .NET. SourceGrid is free
Windows Forms control written entirely in C\#.

VS.NET is one of the world's leading Integrated Development Environment (IDE). With its help, we can do each of the typical tasks related to building an application - writing code, creating user interface, compiling, running and testing, debugging, error tracking, viewing documentation and others [10, 11]. The Microsoft .NET Framework is platform created by Microsoft that provides a programming model, library of classes, Framework Class Library (FCL), and Common Language Runtime (CRL). .NET applications are written in high-level languages (C\#, VB.NET, C ++ / CLI, etc.) and compiled into a platform-independent intermediate language called Common Intermediate Language (CIL). In this work, we used the C\# language. During execution, the CIL code is automatically compiled by CLR for the specific hardware platform and current operating system.

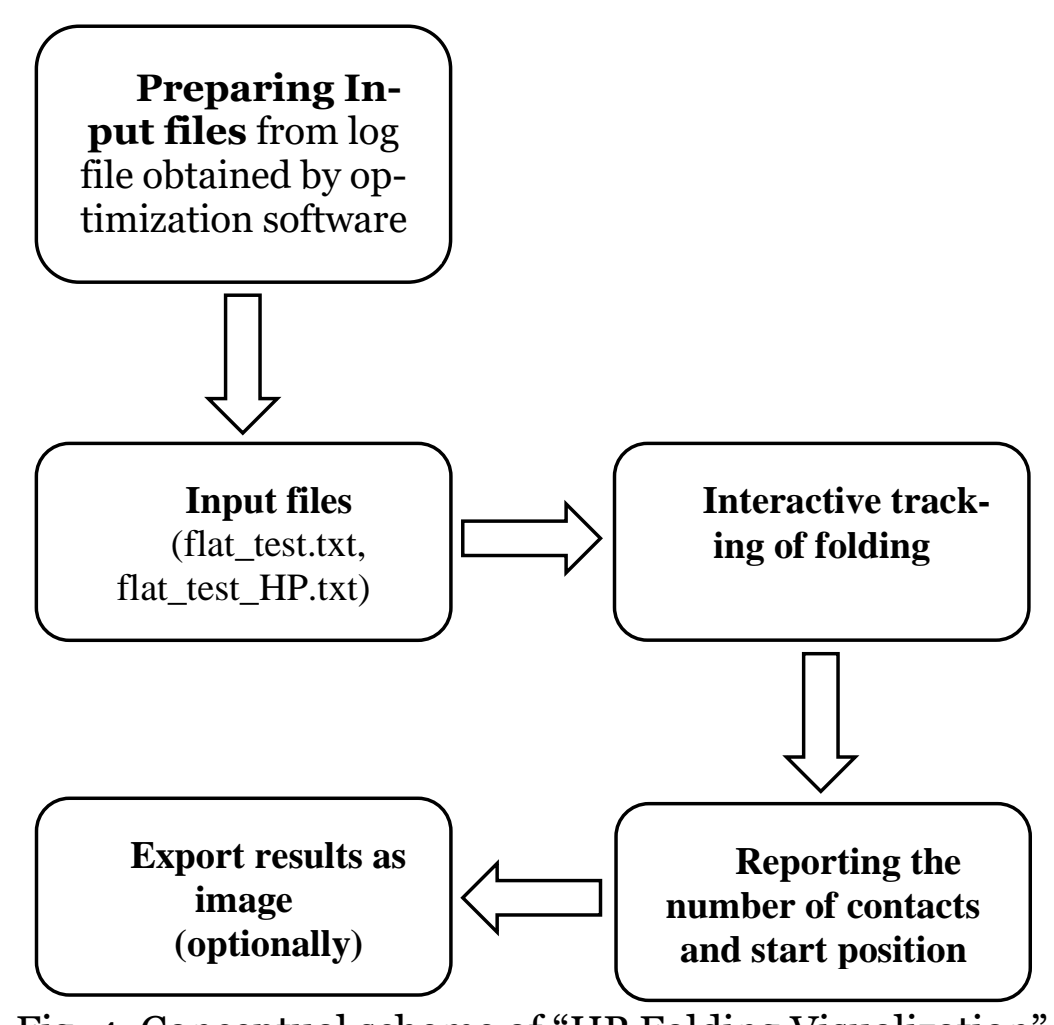

Fig. 4. Conceptual scheme of "HP Folding Visualization" 
Using our software, we can interactively track the folding process (Figure 5), i.e. each movement of the amino acids in the $2 \mathrm{D}$ square lattice, $\mathrm{H}-\mathrm{H}$ contacts are marked as “\#” and colored in red.

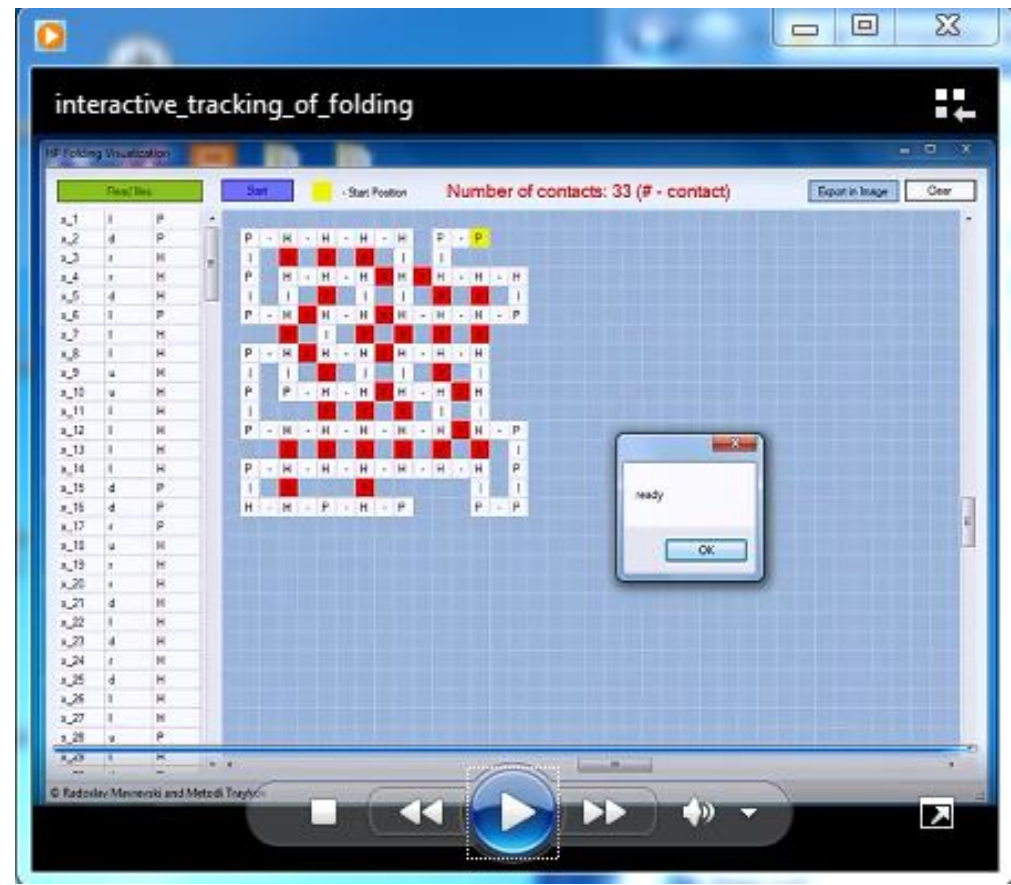

Fig. 5. Interactively track the folding process (video file)

The start position is marked with yellow color. With dash we indicate the adjacent amino acids in the input HP sequence (see Figure 6).

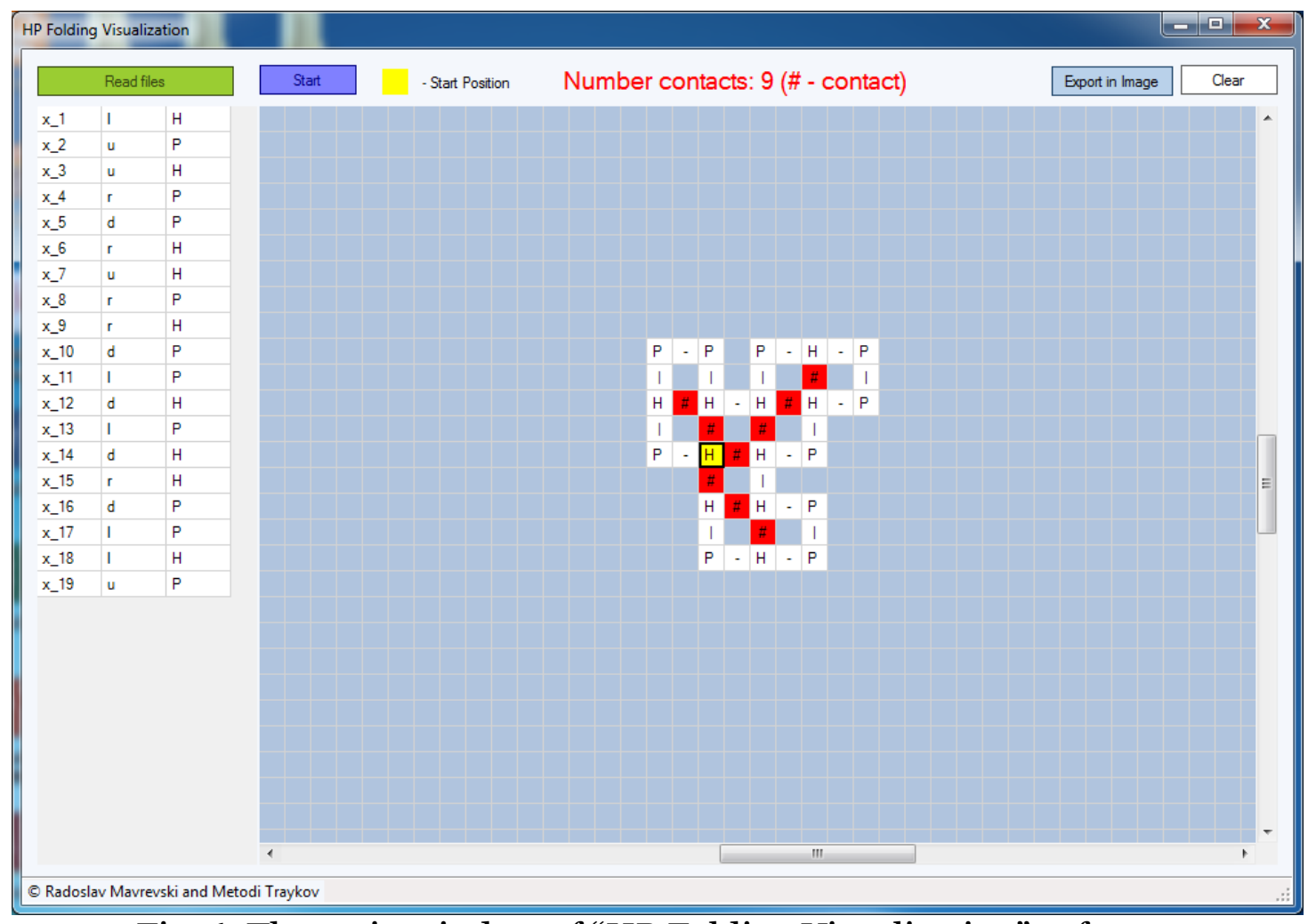

Fig. 6. The main window of "HP Folding Visualization" software 


\section{Results Discussion}

In this article, we described the software "HP Folding Visualization" for 2D visualization of the HP folding developed by us. For our examples, we use output files from CPLEX that are solutions of mathematical models described in .lp files. These output files contain obtained folds for HP sequences with different length [9].

The next figures show obtained results by using "HP Folding Visualization" software.. In Figure 7 we show obtained folds for HP sequences with length:

(a) 20 amino acids - H P H P P H H P H P P H P H H P P H P H;

(b) 24 amino acids - H H P P H P P H P P H P P H P P H P P H P P H H;

(c) 60 amino acids -P P H H H P H H H H H H H H P P P H H H H H H H H H H P H P P P H H H H H H H H H H H H P P P P H H H H H H P H H P H P.

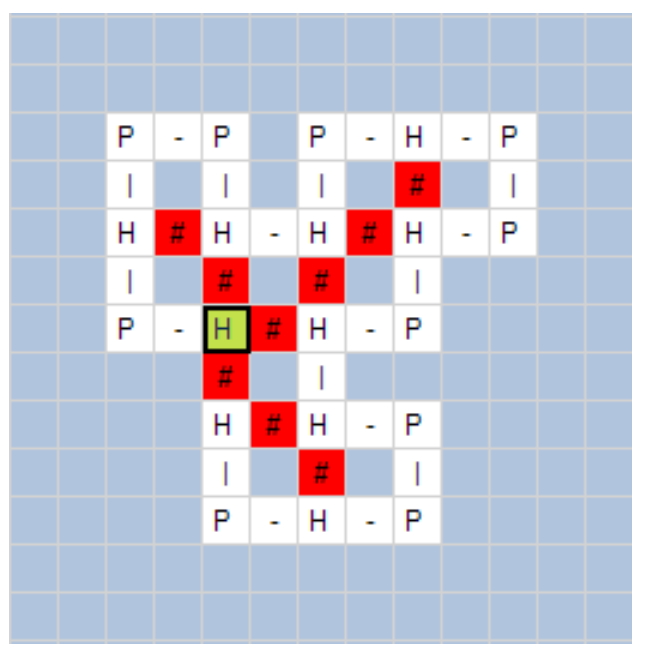

(a)

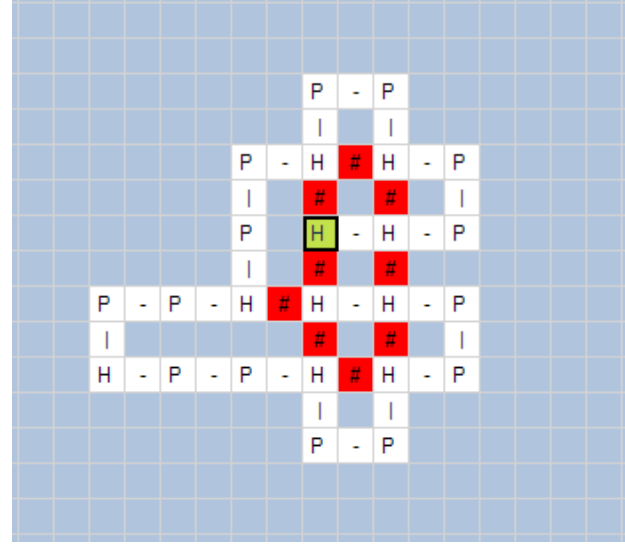

(b)

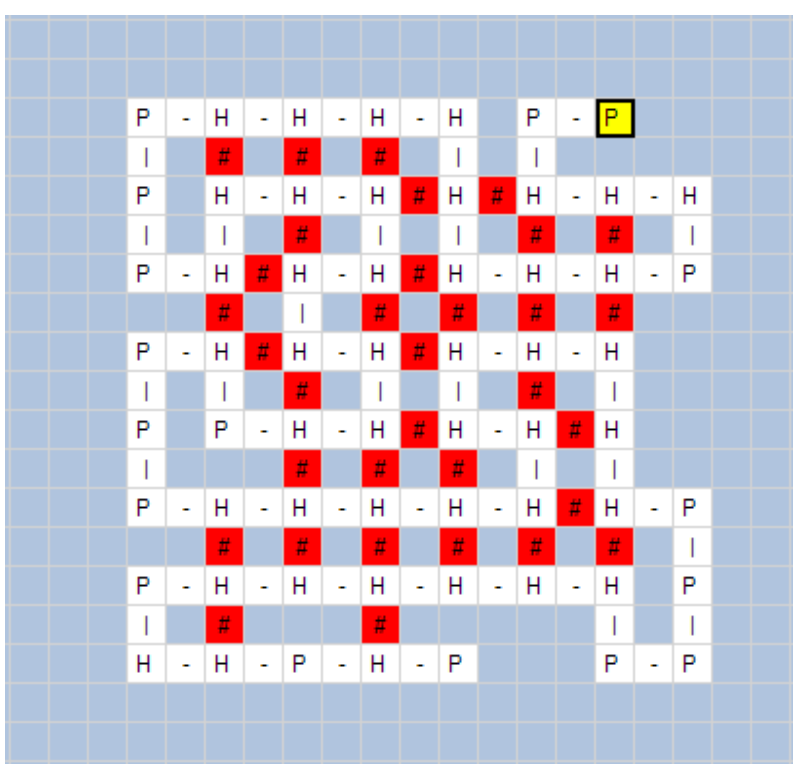

(c)

Fig. 7. Visualization of HP folding by "HP Folding Visualization" software: (a) protein with length 20 , (b) protein with length 24 , (c) protein with length 60.

In the above figures, we can see the $\mathrm{H}$ $\mathrm{H}$ contacts (the contact between $\mathrm{H}$ amino acids that are not adjacent in the protein sequence) and their number.

All data for our input files are obtained using algorithm described by Yanev et. al [9]. This algorithm uses mathematical model (Integer Programming) for $\mathrm{HP}$ model in 2D square lattice and as we said above, we solve this model with MIP solver CPLEX. The CPLEX solution file (.sol or .log) contains solution variables (binary type) (see Figure 1) for the objective function described in 
.lp file, i.e. the input for "HP Folding Visualization" software is file that contains binary variables (number of binary variables is equal to number of amino acids). This is the reason why we cannot compare our software with other bioinformatics visualization tools (e.g. Bioblender (http://www.bioblender.org/), PEPFOLD (http://bioserv.rpbs.univ-parisdiderot.fr/services/PEP-FOLD3/),

PyMOL (https://pymol.org/), ProtSkin (http://www.mcgnmr.mcgill.ca/ProtSk in/) etc.) because they use input files as .pdb, .cif, .xml, etc. To run the input file for "HP Folding Visualization" software in mentioned above bioinformatics visualization tools we need to convert the CPLEX solution file (with binary variables) to .pdb, .cif or xml file that contains specific information (as distances in Ångström etc.) for protein. On the other hand, the bioinformatics visualization tools, like Bioblender and PEP-FOLD, cannot visualize the results obtained by algorithms that use Integer Programming approach (like Yanev et al. algorithm) $[9,12]$. To work correctly most bioinformatics visualization tools need .pdb or FASTA files, protein PDB ID, specific coordination or original amino acid sequence (not HP sequence), i.e. they cannot visualize folds obtained by Integer Programming approach (2D or $3 \mathrm{D}$ lattices HP model) and algorithms as mentioned above.

\section{Conclusions}

The computational experiments were made in simple 2D lattice HP model. This model is used by Yanev et al. to solve HP folding problem, so discussed folds are proven and described in [9]. In particular, our software may be helpful to probe details of folding trajectories and number of contacts in the study of proteins folding. Using our software, we expect to be able to shed light into the nature of these various conformational states. In addition this article can help the students in secondary and higher schools to acquire additional skills in bioinformatics and to learn more about the proteins and their folding.

Ahead of us stands the challenge to use our software together with other methods that efficiently visualize the HP folding in different lattice types.

If you want to use "HP Folding Visualization" software please send us mail on radoslav sm@abv.bg. It will be pleasure for us to provide our software.

\section{References}

1. Gō N, Taketomi H: Respective roles of short- and long-range interactions in protein folding. Proceedings of the National Academy of Sciences of the United States of America 1978, 75:559-563.

2. Dill KA: Theory for the folding and stability of globular proteins. Biochemistry 1985, 24:1501-1509.

3. Lau KF, Dill KA: A lattice statistical mechanics model of the conformational and sequence spaces of proteins. Macromolecules 1989, 22:3986-3997.

4. Istrail, S., and Lam, F. 2009. Combinatorial algorithms for protein folding in lattice models: A survey of mathematical results. Commun. Inf. Syst. 9, 303-346.

5. Dill KA, Bromberg S, Yue K, Fiebig KM, Yee DP, Thomas PD, Chan HS: Principles of protein folding - A perspective from simple exact models. Protein Science 1995, 4:561-602.

6. Thalheim T., Merkle D., Middendorf M. Protein Folding in the HP-Model Solved With a Hybrid Population Based ACO Algorithm. IAENG International Journal of Computer Science 2008, 35:391-300.

7. Shan Y, Arkhipov A, Kim ET, Pan AC, Shaw DE (2013) Transitions to catalytically inactive conformations in EGFR kinase. Proceedings of the National Academy of Sciences of the United States of America 110: 7270-7275. 
8. Reddy AS, Wang L, Singh S, Ling YL, Buchanan L, et al. (2010) Stable and metastable states of human amylin in solution. Biophysical Journal 99: 2208-2216.

9. Yanev N., Traykov M., Milanov P., Yurukov B. Protein Folding Prediction in a Cubic Lattice in HydrophobicPolar Model, Journal of Computational Biology, 2017, 24(5), 412-421

10. Arora, G., Aiaswamy, B., Pandey, N. Microsoft C\# Professional Projects, Portland, United States, Premier Press, 2002.

11. Krishna, P. "Announcing the .NET Framework 4.7.1". .NET Blog. Retrieved 17 October 2017.

12. Yoon, H., 2006. Optimization Approaches to Protein Folding. Georgia: School of Industrial and System Engineering, Institute of Technology 